\title{
Bridging therapy is associated with improved cognitive function after large vessel occlusion stroke - an analysis of the German Stroke Registry
}

Philipp Ettelt ${ }^{1 *+}$, Ilko L. Maier ${ }^{2^{* \dagger}}$ (D), Marlena Schnieder ${ }^{2}$, Mathias Bähr ${ }^{2}$, Daniel Behme ${ }^{3}$, Marios-Nikos Psychogios ${ }^{4}$, Jan Liman ${ }^{2}$ and on behalf of the GSR-ET Collaborators

\begin{abstract}
Background: The targeted use of endovascular therapy (EVT), with or without intravenous thrombolysis (IVT) in acute large cerebral vessel occlusion stroke (LVOS) has been proven to be superior compared to IVT alone. Despite favorable functional outcome, many patients complain about cognitive decline after EVT. If IVT in addition to EVT has positive effects on cognitive function is unclear.

Methods: We analyzed data from the German Stroke Registry (GSR, an open, multicenter and prospective observational study) and compared cognitive function 90 days after index ischemic stroke using MoCA in patients with independent (mRS $\leq 2$ pts) and excellent ( $\mathrm{mRS}=0$ pts) functional outcome receiving combined EVT and IVT $(E V T+$ IVT) vs. EVT alone (EVT-IVT).

Results: Of the 2636 GSR patients, we included 166 patients with mRS $\leq 2$ at 90 days in our analysis. Of these, 103 patients (62\%) received EVT + IVT, 63 patients (38\%) were treated with EVT alone. There was no difference in reperfusion status between groups ( $\mathrm{mTICl} \geq 2 \mathrm{~b}$ in both groups at $95 \%, p=0.65)$. Median MoCA score in the EVT + IVT group was 20 pts. (18-25 IQR) vs. 18 pts. (16-21 IQR) in the EVT-IVT group ( $p=0.014)$. There were more patients with cognitive impairment (defined as MoCA < 26 pts) in the EVT-IVT group (54 patients (86\%)) compared to the $\mathrm{EVT}+\mathrm{IVT}$ group (78 patients (76\%)). EVT + IVT was associated with a higher MoCA score at 90 days (mRS $\leq 2: p=$ $0.033, \mathrm{~B}=2.39 ; \mathrm{mRS}=0: p=0.021, \mathrm{~B}=4.38)$.
\end{abstract}

Conclusions: In Patients with good functional outcome after LVOS, rates of cognitive impairment are lower with combined EVT and IVT compared to EVT alone.

Trial registration: ClinicalTrials.gov Identifier: NCT03356392.

Keywords: Ischemic stroke, LVOS, Intravenous thrombolysis, Mechanical thrombectomy, Cognitive function

\footnotetext{
* Correspondence: philipp.ettelt@stud.uni-goettingen.de; Ilko.Maier@med.uni-

goettingen.de

${ }^{+}$Philipp Ettelt and Ilko L. Maier contributed equally to this work.

${ }^{1}$ Department of Neurology, Allgemeines Krankenhaus Celle, Celle, Germany

2Department of Neurology, University Medicine Göttingen, Göttingen,

Germany

Full list of author information is available at the end of the article
}

(c) The Author(s). 2020 Open Access This article is licensed under a Creative Commons Attribution 4.0 International License, which permits use, sharing, adaptation, distribution and reproduction in any medium or format, as long as you give appropriate credit to the original author(s) and the source, provide a link to the Creative Commons licence, and indicate if changes were made. The images or other third party material in this article are included in the article's Creative Commons licence, unless indicated otherwise in a credit line to the material. If material is not included in the article's Creative Commons licence and your intended use is not permitted by statutory regulation or exceeds the permitted use, you will need to obtain permission directly from the copyright holder. To view a copy of this licence, visit http://creativecommons.org/licenses/by/4.0/. 


\section{Introduction}

Ischemic stroke is one of the most frequent causes of permanent physical disability worldwide [1] and, in addition, is associated with an increasing incidence of cognitive impairment and dementia [2]. Even after good clinical recovery, about $30-50 \%$ of patients complain of cognitive impairment or dementia within the first year after an ischemic stroke [3-7]. In the course, the prevalence of dementia even increases [8] with a broad spectrum of cognitive disturbances, ranging from mild cognitive impairment into a manifest dementia [9-12]. Cognitive impairment not only reduces quality of life, but also increases mortality and health care costs [13]. The exact pathogenesis so far remains unclear. Since both ischemic stroke and cognitive impairment have an increasing prevalence in older age, the exact differentiation, especially of causality, is not easy. Pendlebury et al. could show a significant deterioration of cognitive function associated with an ischemic event in patients with already beginning (age-correlated-physiological) cognitive impairment [14]. Secondary analysis of the Framingham Heart Study conclude that post-stroke worsening in several dimensions of cognition cannot be explained solely by inferior cognitive performance before the stroke event or by concomitant common vascular risk factors [15]. One might assume that ischemic stroke causes a significant loss of cerebral substance with insufficient compensation due to the already aged "senile" brain. The functional "reserve" is lower in old age, which is also reflected in the risk factors for a post-stroke cognitive disorder: Here, in addition to the age (> 65 years) and already known cognitive impairment, cerebral atrophy in the temporal lobe, recurrent ischemic strokes, cardioembolic infarcts and so-called "white matter lesions" are mentioned [16-19].

Early recanalizing treatment of stroke reduces ischemic lesion burden. In addition to the treatment with thrombolysis using intravenous tissue plasminogen activator (rtPA, IVT), endovascular therapy (EVT) has been shown to be the gold standard in the acute phase and significantly improves functional outcome [20-24]. In addition, a recent study by López-Cancio et al. could demonstrate the benefit of EVT versus IVT alone for cognitive outcome parameters in a group of patients with good functional outcome (defined as a score on the modified Rankin Scale (mRS): $\leq 2$ ) [25].

In the acute treatment of stroke IVT is often combined with EVT ("bridging-therapy"). Currently, the advantage of bridging therapy versus non-bridging is controversially discussed [26-29]. Recent studies indicate a possible benefit of bridging therapy in terms of recanalization rate and functional outcome, with no significant increase in the rate of intracranial hemorrhage in the bridging group [30, 31]. An analysis of data from the Virtual International Stroke Trials Archive (VISTA) study suggests that stroke patients may benefit from rtPA treatment in terms of cognition [32]. But it is still unclear whether bridging therapy also has positive effects on cognition compared to EVT alone.

In this study, we investigate the effects of bridging treatment (IVT + EVT) vs EVT alone (EVT-IVT) on cognitive function in patients with LVOS and good outcome.

\section{Material and methods}

Patient population and clinical characteristics

Available data of all patients enrolled in the German Stroke Registry - Endovascular Treatment (GSR-ET 07/ 2015-04/2018; ClinicalTrials.gov Identifier: NCT03356392) were included. The GSR-ET is an ongoing, open-label, prospective, multicenter registry of 25 sites in Germany, collecting consecutive patients undergoing EVT.

\section{Group classification}

Initially, patients with an mRS between 0 and 2 were selected from the entire GSR database in this study. In a next step, only patients with a MoCA-evaluation after 90 days were included for further analysis. A Bridging$($ EVT + IVT) and a Non-Bridging group (EVT-IVT) were compared. Subsequently, subgroups were formed for comparison, which were analyzed separately. This includes a group with a mRS of 0 points, as well as a subgroup formation by stroke-localization (right vs. left and anterior vs. posterior cerebral circulation).

To further differentiate the cognitive impairment (CI), patient groups were formed on the basis of the 90-day MoCA scores (0-9 pts.: severe CI; 10-17 pts.: moderate CI; $18-25$ pts.: mild CI; $>25$ pts. No CI). Although this classification is currently not based on scientific evidence, the homepage offers this classification as a possible graduation of the severity of a CI [33].

\section{Statistical analysis}

The direct group comparison was done by descriptive statistics. Categorical sizes were given with mean and standard deviation, as well as absolute frequencies. Continuous variables were given by median, quartiles, minimum and maximum. Comparative test procedures between intervention and control groups were performed by chi-square test and non-parametric method (Mann-Whitney U test), depending on variable. Missing values in variables of the data set were determined with an in-depth data analysis. For continuous and categorical independent variables with more than $10 \%$ missing values, the multiple imputation feature implemented in SPSS was used to calculate the missing values using a regression model [34]. This method was used to 
counteract the bias of the result by complete-case analysis and to increase the validity of the study [35-37]. Potential confounders were filtered out by univariate pre-testing, with a $p$-value $<0.3$ being considered predictive of the outcome "cognitive impairment". For the continuous endpoint MoCA-value after 90 days, a linear regression model was performed. Finally, the two groups with possible confounders were further processed in a multivariate logistic regression model. All calculations were based on a $5 \%$ significance level.

Statistical analysis was performed using SPSS (version 26, IBM Corporation, Armonk, New York, USA), written with Word (Microsoft Corporation, Redmond, Washington, USA). Graphics were created using Excel (ibid).

\section{Results}

\section{Baseline-characteristics}

From the 2636 patients included in the GSR the 90-day MoCA had been recorded in 215 (8.1\%) patients. Of these, $166(77.2 \%)$ patients had an independent functional outcome with a 90-day $\mathrm{mRS} \leq 2$. In this group 103 (62\%) patients were treated with EVT + IVT, 63 (38\%) received an EVT alone. These patients most likely did not receive EVT due to various contraindications for IVT $(24(38 \%)$ patients had an unclear or exceeded time window with already beginning infarct signs in the imaging, 39 (62\%) had an active anticoagulation, severe comorbidities as contraindication for IVT or recent surgery). The baseline characteristics of both groups are summarized in Table 1 . In most cases stroke was located in the anterior cerebral circulation $(n=146,88 \%)$; the remaining $20(12 \%)$ patients had vertebrobasilar strokes. There was a significant shorter median symptom onset to admission time in patients with EVT + IVT compared to in the EVT-IVT group (69 $\mathrm{min}$, IQR $40-171 \mathrm{~min}$ vs 205 IQR $73-508 \mathrm{~min}$ in the EVT-IVT group, $p<0.001$ ). The proportion of smokers $(37.1 \%$ vs. $27.3 \%, p=0.19)$ and patients with anticoagulation $(28,6 \%$ vs. $0 \%, p<$ 0.001) were higher in the EVT-IVT group compared to the EVT + IVT group. Functional impairment, quantified by the National Institute of Health Stroke Scale (NIHSS) on admission was more pronounced in the EVT + IVT group (12 pts., IQR 7-16 pts., vs. 10 pts., IQR 5-15 pts., $p=0.14$ ) as well as the ASPECT score for the assessment of early infarct signs, which was also higher in the EVT + IVT group (9 pts., IQR $8-10$ pts. vs. 8 pts., IQR 7-10, $p=0.01$ ). There was no significant difference in groin to reperfusion time in both groups (37 min, IQR 22,5-56 min in the EVT + IVT-group and 34 min, IQR 26-55 in the EVT-IVT group; $p=0.922$ ). At discharge, both groups showed mild symptoms with a median NIHSS of 2 points $(p=0.24)$.

A subgroup of $54(33.7 \%)$ patients had no stroke associated functional impairment corresponding to a 90-day mRS of 0 pts. The baseline characteristics of these patients are given in supplementary Table 1 . This subgroup showed differences in gender distribution with a lower proportion of male patients in the EVT + IVT group compared to the EVT-IVT group (38.5\% vs. $66.7 \%, p=0.06)$. Again, there was a significant difference between the two groups in terms of median time between symptom onset and admission (82 min, IQR 45-236 $\mathrm{min}$ in the EVT + IVT group, 156 min, IQR 73-553 min in the EVT-IVT group, $p<$ 0.001). Patients with bridging therapy also had a higher NIHSS on admission compared to EVT-IVT patients (NIHSS median 11 pts., IQR $7-15$ in the EVT + IVT group vs. 6 pts., IQR 4-12 in the EVTIVT group, $p=0.057$ ), while the NIHSS was slightly higher at discharge in the EVT-IVT group (1 pt., IQR $0-2$ in the EVT-IVT and 0 pts., IQR $0-2$ in the EVT + IVT group, $p=0.101)$. Again, the proportion of anticoagulated patients in the EVT-IVT group was higher $(33.3 \%$ vs. $0 \%, p<0.001)$.

\section{MoCA-score at day 90}

For patients with a 90-day $m R S \leq 2$, there was a significant difference in the MoCA scores between the EVT + IVT and EVT-IVT group with a median MoCA score in the EVT + IVT group of 20 points (IQR 1825 pts) vs 18 points (IQR 16-21 pts) in the EVT-IVT group $(p=0.014)$. For patients with excellent functional outcome $(m R S=0)$, we also found a significantly higher MoCA score in the EVT + IVT group (21 points (IQR $18-29$ pts) vs 19 points (IQR $17-21$ pts), $p=0.018$ ).

After the adjustment for sex, age, NIHSS, symptomonset to admission-time, ASPECTS, vascular-risk factors and baseline medication a significant association between EVT + IVT and higher MoCA-scores after 90 days persisted both for patients with independent- as well as excellent functional outcome $(\mathrm{mRS} \leq 2 \mathrm{:} \mathrm{B}=2.39$, 95\%$\mathrm{CI}=0.20-4.58, p=0.033 ; \quad \mathrm{mRS}=0: \mathrm{B}=4.38,95 \%-\mathrm{CI}=$ $0.67-8.08, p=0.021$ ) (Table 2). This difference was independent concerning reperfusion status after endovascular treatment since $95 \%$ reached $\mathrm{mTICI} \geq 2 \mathrm{~b}$ in both groups $(p=0.65)$.

After correction for relevant confounders (supplementary Table 2), patients with strokes in the anterior circulation receiving combined EVT and IVT tended to show a higher MoCA-score compared to the EVT-IVT group $(\mathrm{B}=2.29 ; p=0.061$; median MoCA 20 pts. (IQR 17-24 pts) vs 18 points (IQR $16-21$ pts)). There were no significant differences in MoCA-scores between both groups in patients with strokes in the posterior circulation (median MoCA 21 pts., IQR $18-28$ in the EVT + IVT vs 20 pts., IQR 14-27, in the EVT-IVT group; $\mathrm{B}=$ 2.12; $p=0.603$ ) (supplementary Table 3). 
Table 1 Baseline Characteristics MRS $\leq 2$

\begin{tabular}{|c|c|c|c|}
\hline Variable & $\begin{array}{l}\text { Bridging } \\
(\boldsymbol{n}=103)\end{array}$ & $\begin{array}{l}\text { Non-Bridging } \\
(\boldsymbol{n}=63)\end{array}$ & $\begin{array}{l}\boldsymbol{p}^{-} \\
\text {value }\end{array}$ \\
\hline $\operatorname{Sex}(n, \%)$ & & & 0.29 \\
\hline Male & $55(53.4)$ & $39(61.9)$ & \\
\hline Female & $48(46.6)$ & $24(38.1)$ & \\
\hline Age $($ mean $\pm S D)$ & $67( \pm 14.28)$ & $69( \pm 11.18)$ & 0.29 \\
\hline \multicolumn{4}{|l|}{ Vasc. risk-factors $(n, \%)$} \\
\hline Art. hypertension & $69(67)$ & $45(71.4)$ & 0.55 \\
\hline Smoking & $27(27.3)$ & $23(37.1)$ & 0.19 \\
\hline Diabetes & $17(16.5)$ & $14(22.2)$ & 0.36 \\
\hline Dyslipidemia & $39(37.9)$ & $23(36.5)$ & 0.86 \\
\hline Atrial fibrillation & $25(24.3)$ & $21(33.3)$ & 0.21 \\
\hline \multicolumn{4}{|l|}{ Baseline medication ( $n, \%)$} \\
\hline Aspirin & $28(28.9)$ & $12(20)$ & 0.22 \\
\hline Clopidogrel & $3(3.1)$ & $2(3.3)$ & 0.93 \\
\hline Anticoagulation & $0(0)$ & 18 (28.6) & $<0.001$ \\
\hline NIHSS at admission (median points, IQR) & $12(7-16)$ & $10(5-15)$ & 0.14 \\
\hline Symptom-onset to admission (median time, IQR) & $69(40-171)$ & $205(73-508)$ & $<0.001$ \\
\hline ASPECTS (median points, IQR) & $9(8-10)$ & $8(7-10)$ & 0.01 \\
\hline General anesthesia (n, \%) & $68(66)$ & $41(65)$ & 0.16 \\
\hline Treatment adverse event ( $n, \%)$ & $12(11)$ & $8(12)$ & 0.72 \\
\hline NIHSS at discharge (median points, IQR) & $2(0-4)$ & $2(1-3)$ & 0.24 \\
\hline $\mathrm{mTICl} \geq 2 \mathrm{~b}$ after EVT $(\mathrm{n}, \%)$ & $98(95)$ & $60(95)$ & 0.65 \\
\hline \multicolumn{4}{|l|}{ Stroke etiology (n, \%) } \\
\hline Large-artery atherosclerosis & $22(21)$ & $16(25)$ & 0.57 \\
\hline Cardioembolism & $37(36)$ & $27(43)$ & 0.41 \\
\hline Small-vessel occlusion & $0(0)$ & $1(1)$ & 0.38 \\
\hline Stroke of other determined etiology & $9(9)$ & $2(3)$ & 0.21 \\
\hline Stroke of undetermined etiology & $23(22)$ & $15(24)$ & 0.85 \\
\hline \multicolumn{4}{|l|}{ Stroke localization ( $\mathrm{n}, \%)$} \\
\hline Right hemispheric stroke & $56(54)$ & $29(46)$ & 0.44 \\
\hline Left hemispheric stroke & $34(33)$ & $27(43)$ & 0.44 \\
\hline Vertebrobasilar stroke & $13(13)$ & $7(11)$ & 0.99 \\
\hline A. cerebri anterior & $1(1)$ & $0(0)$ & 0.99 \\
\hline A. cerebri media M1 proximal & $33(32)$ & $28(44)$ & 0.14 \\
\hline A. cerebri media M1 distal & $26(25)$ & $14(22)$ & 0.71 \\
\hline A. cerebri media M2 & $23(22)$ & $13(21)$ & 0.85 \\
\hline A. carotis interna $T$ & $10(10)$ & $5(8)$ & 0.79 \\
\hline A. carotis interna extracranial & $2(2)$ & $2(3)$ & 0.64 \\
\hline
\end{tabular}

$S D$ standart deviation, IQR interquartile range

\section{Cognitive impairment}

Overall, 133 of the 166 patients included in the analysis (80\%) showed cognitive impairment (defined as a MoCA-score $<26$ points) 90 days after the index event. The rate was $76 \%$ in the EVT + IVT and $86 \%$ in the EVT-IVT group. However, this difference was not statistically significant after correction for possible confounders $(p=0.225$; OR: 2.27$)$. In the group with excellent functional outcome $(\mathrm{mRS}=0)$ the prevalence of cognitive impairment was significantly lower (66\%) in the EVT + IVT group, compared to the EVT-IVT group (93\%; $p=0.034 ;$ OR: $23.15 ;$ suppl. Table 1$)$. In the 
Table 2 Influence of variables on the 90d MoCA value

\begin{tabular}{|c|c|c|c|c|}
\hline \multirow[t]{2}{*}{ Variable } & \multicolumn{2}{|c|}{ Single linear regression } & \multicolumn{2}{|c|}{ Multiple linear regression } \\
\hline & $\mathrm{B}(95 \% \mathrm{Cl})$ & $\boldsymbol{p}$-value & B (95\% Cl) & $\boldsymbol{p}$-value \\
\hline \multicolumn{5}{|l|}{ MRS $\leq 2$} \\
\hline Bridging-therapy & $2.01(0.4-3.62)$ & 0.015 & $2.39(0.20-4.58)$ & 0.033 \\
\hline Sex & & & $0.52(-1.08-2.12)$ & 0.526 \\
\hline Age & & & $-0.11(-0.17--0.04)$ & 0.001 \\
\hline Symptom-onset to admission & & & $0.00(-0.003-0.003)$ & 0.842 \\
\hline NIHSS at admission & & & $-0.04(-0.17-0.09)$ & 0.551 \\
\hline Smoking & & & $-1.91(-3.71--0.11)$ & 0.038 \\
\hline Atrial fibrillation & & & $-0.60(-2.71-1.51)$ & 0.576 \\
\hline Aspirin premedication & & & $-0.5(-2.48-1.49)$ & 0.623 \\
\hline Anticoagulation & & & $1.91(-1.23-5.05)$ & 0.232 \\
\hline ASPECT-Score & & & $-0.25(-0.94-0.43)$ & 0.46 \\
\hline NIHSS at discharge & & & $-0.09(-0.29-0.12)$ & 0.405 \\
\hline \multicolumn{5}{|l|}{ MRS $=0$} \\
\hline Bridging-therapy & $3.55(0.78-6.31)$ & 0.013 & $4.38(0.67-8.08)$ & 0.021 \\
\hline Sex & & & $0.58(-2.1-3.26)$ & 0.669 \\
\hline Age & & & $-0.07(-0.21-0.07)$ & 0.319 \\
\hline Symptom-onset to admission & & & $-0.003(-0.76-1.07)$ & 0.721 \\
\hline NIHSS at admission & & & $-0.06(-0.24-0.13)$ & 0.56 \\
\hline Smoking & & & $0.80(-2.50-4.11)$ & 0.63 \\
\hline Dyslipidemia & & & $2.49(-0.28-5.27)$ & 0.078 \\
\hline Aspirin premedication & & & $-2.28(-5.41-0.86)$ & 0.154 \\
\hline Anticoagulation & & & $-0.49(-7.32-6.34)$ & 0.881 \\
\hline NIHSS at discharge & & & $0.16(-0.76-1.07)$ & 0.721 \\
\hline
\end{tabular}

Cl Confidence Interval

subgroup analysis on individual examination of the stroke localization, there was no significant difference between the prevalence of cognitive impairment between the EVT + IVT and EVT-IVT group (suppl. Table 4).

\section{Severity of cognitive-impairment}

Rates of moderate and severe cognitive impairment were significantly higher in the EVT-IVT (25 of 63 patients; $39.6 \%$ ) versus the EVT + IVT group (25 of 103 patients, $24 \% ; p=0.040$, OR 3.38). In patients with $\mathrm{mRS}=0$, there also was a significant higher risk for moderate to severe cognitive impairment at 90 days with a prevalence of $13 \%$ in the EVT + IVT- and 33\% in the EVT-IVT group ( $p=0.035$, OR: 0.08) (Table 3).

\section{Discussion}

In the present study, we found lower rates of cognitive impairment in LVOS-patients treated with EVT + IVT compared to patients with EVT alone. EVT + IVT patients performed better in the 90-day MoCA compared to patients treated with EVT alone, even though the initial neurological deficits were more pronounced in the
EVT + IVT group. Concerning the similar reperfusion rates in both groups and the correction for symptom onset to admission time in our analysis, IVT seems to have a positive effect not only on functional outcome-, but also on cognition after stroke. In particular, IVT seems to have the highest effect in patients with excellent functional outcome. The effect of rtPA on cognition has so far been poorly understood, and the few post-stroke studies with cognitive endpoints showed heterogeneous results [38]. This is probably due to the different test methods used. At least until now, a benefit of the rtPA therapy with regard to visuoconstrictive abilities could be recognized [39].

Comparison of baseline characteristics between the EVT + IVT and the EVT-IVT group showed a significant difference in onset-to-admission times. In the EVT + IVT group $25 \%$ of patients have been treated within 40 min after symptom-onset, which, considering the findings of this study, underlines the importance to minimize onset-to-admission times to achieve not only favorable functional-, but also favorable cognitive outcomes. 
Table 3 Severity of Cognitive Impairment

\begin{tabular}{|c|c|c|c|c|}
\hline & $\begin{array}{l}\text { Bridging-group } \\
(n, \%)\end{array}$ & $\begin{array}{l}\text { Non-Bridging group } \\
(\mathrm{n}, \%)\end{array}$ & OR $(95 \% \mathrm{Cl})$ & $\begin{array}{l}p- \\
\text { value }\end{array}$ \\
\hline \multicolumn{5}{|l|}{$\mathrm{mRS} \leq 2$} \\
\hline No Cognitive Impairment & $25(24.3)$ & $9(14.3)$ & $0.52(0.23-1.20)$ & 0.126 \\
\hline Mild Cognitive Impairment & $53(51.4)$ & $29(46)$ & $0.66(0.27-1.60)$ & 0.355 \\
\hline Moderate Cognitive Impairment & $25(24.3)$ & $24(38.1)$ & $0.38(0.15-0.97)$ & 0.042 \\
\hline Severe Cognitive Impairment & $0(0)$ & $1(1.5)$ & - & - \\
\hline \multicolumn{5}{|l|}{$\mathrm{mRS}=0$} \\
\hline No Cognitive Impairment & $13(33.3)$ & $1(6.6)$ & $0.14(0.02-1.21)$ & 0.074 \\
\hline Mild Cognitive Impairment & $21(53.8)$ & $9(60)$ & $0.18(0.02-1.59)$ & 0.122 \\
\hline Moderate Cognitive Impairment & $5(12.8)$ & $5(33.3)$ & $0.08(0.01-0.83)$ & 0.035 \\
\hline Severe Cognitive Impairment & $0(0)$ & $0(0)$ & - & - \\
\hline
\end{tabular}

Cognitive Impairment (Cl): No Cl: MoCA $\geq 26$ pts., Mild Cl: MoCA > 17 pts., Moderate Cl: MoCA > 10 pts., Severe Cl: MoCA < 10 pts.

In our study, we found a high rate of $\mathrm{CI}$ after 90 days in general (80\%), which is well above the expected prevalence in other studies [3, 4, 7]. A selection-bias could occur due to the limited group size in this study and therefore could limit the generalizability of our data, as only around $8 \%$ of patients included in the GSR received cognitive testing. This low rate is most likely to be explained by the mere necessity of a personal follow-up visit, which certainly results in a high proportion of drop-outs. Furthermore, patients with post stroke aphasia or pre-existing CI could not be entirely excluded from the study population. Both pre-stroke cognitive impairment and post stroke aphasia are major confounders concerning post-stroke cognitive function and lack of correction for these factors represents a major limitation of our study. However, one could argue that it is likely that only patients underwent MoCA-testing being judged eligible to perform the tasks by the rater and that patients with significant aphasia and pre-stroke cognitive impairment should not have been rated as having a 90 days $m R S \leq 2$. In addition, the time span of 90 days between cognitive testing and index event is very tight for the assessment of a stroke-related CI. Other studies suggest an interval of at least 6 months to even speak of post-stroke dementia [12]. However, an increased incidence of $\mathrm{CI}$ in the first period following a stroke or even TIA has been described in other studies [6, 8, 40-42]. Another bias in this study might occur due to the lack of cognitive testing at baseline, so no definitive statement on the incidence of $\mathrm{CI}$, but only on prevalence in the course after stroke event can be made. Nonetheless, the prevalence remains considerable, bearing in mind that it refers to a patient population with good functional outcome.

A particular advantage in terms of cognition at different stroke localization could not be demonstrated. At least we could show that EVT + IVT did not yield any difference in terms of cognitive outcome in patients with stroke located in the posterior cerebral circulation. This also seems understandable when considering the brain region which is provided for by the vertebrobasilar vessels. Especially the neuropsychological and higher cognitive performance, which are queried by the testing via MoCA, are supplied with blood by the anterior cerebral circulation.

The conspicuous link between poorer MoCA testing in smokers in this study has already been observed in larger studies [43, 44] and underlines the importance of nicotine abstinence as a modifiable risk factor in the post-stroke situation. Furthermore, the obvious correlation between higher age and worse performance in the MoCA test is understandable, as the prevalence of $\mathrm{CI}$ increases in older age $[45,46]$.

The classification of $\mathrm{CI}$ into different categories (none, mild, moderate and severe $\mathrm{CI}$ ) based on the MoCA score has yet not been validated in any study. Thus, this classification should be interpreted with an appropriate restraint. Further studies to evaluate a possible classification of $\mathrm{CI}$ after stroke with corresponding cutoff values in MoCA are desirable. In spite of these facts, considering the distribution of points in the individual groups, it is noticeable that significantly inferior cognitive performance (defined as a MoCA value below 17 points) occurs less frequently in the EVT + IVT than in the EVT-IVT group. This should be taken into account in the discussion about the potential benefits of a bridging therapy in the acute treatment of LVOS.

Our study is limited by various factors. On the one hand, the lack of cognitive baseline testing means that no real statement can be made about the incidence of cognitive disorders after mechanical thrombectomy. In addition, patients with pre-existing cognitive impairment or even dementia could distort the overall result. On the other hand, a further measurement using the MoCA-test 
in the long term would have been desirable to assess the development of cognitive impairments. In future studies, in addition to evaluating cognitive status before enrollment (e.g. by means of a simple questionnaire like the Informant Questionaire for Cognitive Decline in the Elderly IQCODE [47]), a simple follow-up should be considered to minimize the drop-out rate. An improvement in data collection could possibly be achieved by using the telephone version of the MoCA (t-MoCA), which has also been validated in clinical studies and described as very sensitive $[48,49]$. In addition to the major confounders being accounted for in the analysis of this study, there are many other factors influencing cognitive function after stroke like history of previous stroke, history of pre-stroke cognitive decline and postprocedural complications. In this study, it was not possible to take these factors into account, representing a major limitation. Another limitation of this study, besides the low number of cases and possible drop-out rate with the risk of selection-bias, is the retrospective, non-randomized study design. In contrast, data was collected prospectively and with a face-to-face interview in the included patients and were collected in multiple large thrombectomy-centers throughout Germany, representing reliable real word data.

\section{Conclusion}

Our data point to a possible benefit of bridging therapy in the controversy between bridging and non-bridging approaches. Even if the median MoCA value differs only by 2 points between the two groups after 90 days, at least this indicates a trend, which should be further verified in larger-scaled, prospective designed studies. In addition, our findings highlight the importance of routine cognitive testing in patients with favourable outcome after LVOS.

\section{Supplementary information}

Supplementary information accompanies this paper at https://doi.org/10. 1186/s42466-020-00079-9.

Additional file 1 Suppl. Table 1. Influence of variables on Cognitive Impairment after 90 days.

Additional file 2 Suppl. Table 2. Relevant subgroup differencies. Additional file $\mathbf{3}$ Suppl. Table $\mathbf{3}$. Influence of variables in the subgroups on the 90d MoCA value.

Additional file 4 Suppl. Table 4 . Influence of variables on Cognitive Impairment (MoCA $<26$ pts) after 90 days.

\section{Abbreviations}

ASPECTS: Alberta stroke programme early CT score; Cl: Cognitive Impairment; EVT: Endovascular therapy; GSR: German Stroke Registry; IQR: Interquartile range; IVT: Intravenous thrombolysis; LVOS: Large cerebral vessel occlusion stroke; MoCA: Montreal Cognitive Assessment; mRS: Modified Rankin Scale; MTICI: Modified treatment in cerebral ischemia score; NIHSS: National Institutes of Health Stroke Scale; OR: Odds Ratio; rtPA: Recombinant tissue plasminogen activator; VISTA: Virtual International Stroke Trials Archive

\section{Acknowledgements}

Not applicable.

\section{Authors' contributions}

Philipp Ettelt analyzed raw data, performed statistics, drafted and finalized the manuscript and approved the manuscript before submission. Ilko L.

Maier: designed the study and was involved in the acquisition of the data, drafted and finalized the manuscript and approved the manuscript before submission. Marlena Schnieder: was involved in the acquisition of the data and approved the manuscript before submission. Mathias Bähr: contributed to the manuscript and approved the manuscript before submission. Daniel Behme: contributed to the manuscript, was involved in the acquisition of the data and approved the manuscript before submission. Marios-Nikos Psychogios: contributed to the manuscript, was involved in the acquisition of the data and approved the manuscript before submission. Jan Liman: contributed to the manuscript and approved the manuscript before submission. Patient data was collected by the GSR-ET comittee.

\section{Funding}

This research received no specific grant from any funding agency in the public, commercial, or not-for-profit sectors.

\section{Availability of data and materials}

The data that support the findings of this study are available from the GSRET Collaborators but restrictions apply to the availability of these data, which were used under license for the current study, and so are not publicly available. Data are however available from the authors upon reasonable request and with permission of the GSR-ET steering committee.

Ethics approval and consent to participate

The study was approved by the institutional review board (ethics commission of the university medicine Göttingen; 16/2/16).

Consent for publication

Not applicable.

Competing interests

The authors declare that they have no competing interests.

\section{Author details}

${ }^{1}$ Department of Neurology, Allgemeines Krankenhaus Celle, Celle, Germany. 2Department of Neurology, University Medicine Göttingen, Göttingen, Germany. ${ }^{3}$ Department of Neuroradiology, University Medicine Göttingen, Göttingen, Germany. ${ }^{4}$ Department of Neuroradiology, Universitätsspital Basel, Basel, Switzerland.

Received: 14 February 2020 Accepted: 13 March 2020

Published online: 27 July 2020

\section{References}

1. Feigin, V. L., et al. (2015). Update on the global burden of ischemic and hemorrhagic stroke in 1990-2013: The GBD 2013 study. Neuroepidemiology, 45(3), 161-176. https://doi.org/10.1159/000441085.

2. Pendlebury, S. T., \& Rothwell, P. M. (2019). Incidence and prevalence of dementia associated with transient ischaemic attack and stroke: Analysis of the population-based Oxford vascular study. Lancet Neurology, 18(3), 248258. https://doi.org/10.1016/S1474-4422(18)30442-3.

3. Sexton, E., et al. (2019). Systematic review and meta-analysis of the prevalence of cognitive impairment no dementia in the first year poststroke. European Stroke Journal, 4(2), 160-171. https://doi.org/10.1177/ 2396987318825484

4. Mellon, L., et al. (2015). Cognitive impairment six months after ischaemic stroke: a profile from the ASPIRE-S study. BMC Neurology, 15(1), 31. https:// doi.org/10.1186/s12883-015-0288-2.

5. Abdel, D., Rudd, A. G., \& Wolfe Charles, D. A. (2013). Prevalence of Poststroke cognitive impairment. Stroke, 44(1), 138-145. https://doi.org/10.1161/ STROKEAHA.112.670844.

6. Jokinen, $H_{\text {., }}$ et al. (2015). Post-stroke cognitive impairment is common even after successful clinical recovery. European Journal of Neurology, 22(9), 1288 1294. https://doi.org/10.1111/ene.12743. 
7. Tatemichi, T. K., Desmond, D. W., Stern, Y., Paik, M., Sano, M., \& Bagiella, E. (1994). Cognitive impairment after stroke: Frequency, patterns, and relationship to functional abilities. Journal of Neurology, Neurosurgery, and Psychiatry, 57(2), 202-207. https://doi.org/10.1136/jnnp.57.2.202.

8. del Ser, T., et al. (2005). Evolution of cognitive impairment after stroke and risk factors for delayed progression. Stroke, 36(12), 2670-2675. https://doi. org/10.1161/01.STR.0000189626.71033.35.

9. Dichgans, M. (2019). Dementia risk after transient ischaemic attack and stroke. The Lancet Neurology, 18(3), 223-225. https://doi.org/10.1016/S1474 4422(18)30497-6.

10. Ivan, C. S., et al. (2004). Dementia After Stroke. Stroke, 35(6), 1264-1268. https://doi.org/10.1161/01.STR.0000127810.92616.78.

11. Narasimhalu, K., et al. (2009). Severity of CIND and MCI predict incidence of dementia in an ischemic stroke cohort. Neurology, 73(22), 1866-1872. https://doi.org/10.1212/WNL.0b013e3181c3fcb7.

12. Mijajlović, M. D., et al. (2017). Post-stroke dementia - a comprehensive review. BMC Medicine, 15. https://doi.org/10.1186/s12916-017-0779-7.

13. Patel, M. D., Coshall, C., Rudd, A. G., \& Wolfe, C. D. A. (2002). Cognitive impairment after stroke: Clinical determinants and its associations with long-term stroke outcomes. Journal of the American Geriatrics Society, 50(4), 700-706. https://doi.org/10.1046/j.1532-5415.2002.50165.x

14. Pendlebury, S. T., \& Rothwell, P. M. (2009). Prevalence, incidence, and factors associated with pre-stroke and post-stroke dementia: A systematic review and meta-analysis. The Lancet Neurology, 8(11), 1006-1018. https://doi.org/ 10.1016/S1474-4422(09)70236-4.

15. Weinstein, G., et al. (2014). Cognitive Performance after Stroke - The Framingham Heart Study. International Journal of Stroke, 9(0 0), 48-54. https://doi.org/10.1111/ijs.12275.

16. Levine, D. A., et al. (2018). Risk factors for post-stroke cognitive decline: The REGARDS study. Stroke, 49(4), 987-994. https://doi.org/10.1161/STROKEAHA. 117.018529.

17. Li, J., Zhao, Y., \& Mao, J. (2017). Association between the extent of white matter damage and early cognitive impairment following acute ischemic stroke. Experimental and Therapeutic Medicine, 13. https://doi.org/10.3892/ etm.2017.4035.

18. Sachdev, P. S., Chen, X., Brodaty, H., Thompson, C., Altendorf, A., \& Wen, W. (2009). The determinants and longitudinal course of post-stroke mild cognitive impairment. Journal of the International Neuropsychological Society, 15(6), 915-923. https://doi.org/10.1017/S1355617709990579.

19. Mok, V. C. T., Lam, B. Y. K., Wong, A., Ko, H., Markus, H. S., \& Wong, L. K. S. (2017). Early-onset and delayed-onset poststroke dementia - revisiting the mechanisms. Nature Reviews. Neurology, 13(3), 148-159. https://doi.org/10. 1038/nrneurol.2017.16

20. Goyal, M., et al. (2015). Randomized assessment of rapid endovascular treatment of ischemic stroke. The New England Journal of Medicine, 372(11), 1019-1030. https://doi.org/10.1056/NEJMoa1414905.

21. Saver, J. L., et al. (2015). Stent-retriever thrombectomy after intravenous t-PA vs. t-PA alone in stroke. The New England Journal of Medicine, 372(24), 22852295. https://doi.org/10.1056/NEJMoa1415061.

22. Jovin, T. G., et al. (2015). Thrombectomy within 8 hours after symptom onset in ischemic stroke. The New England Journal of Medicine, 372(24), 2296-2306. https://doi.org/10.1056/NEJMoa1503780.

23. Berkhemer, O. A., et al. (2014). A Randomized Trial of Intraarterial Treatment for Acute Ischemic Stroke. https://doi.org/10.1056/NEJMoa1411587 Online]. Available: https://www.nejm.org/doi/10.1056/NEJMoa1411587. [Accessed: 03 Sep 2019].

24. Campbell, B. C. V., et al. (2015). Endovascular therapy for ischemic stroke with perfusion-imaging selection. https://doi.org/10.1056/NEJMoa1414792 [Online]. Available: https://www.nejm.org/doi/10.1056/NEJMoa1414792. [Accessed: 03 Sep 2019].

25. López-Cancio, E., et al. (2017). Endovascular treatment improves cognition after stroke. Neurology, 88(3), 245-251. https://doi.org/10.1212/WNL. 0000000000003517.

26. Choi, J. H., Im, S. H., Lee, K. J., Koo, J. S., Kim, B. S., \& Shin, Y. S. (2018) Comparison of outcomes after mechanical thrombectomy alone or combined with intravenous thrombolysis and mechanical thrombectomy for patients with acute ischemic stroke due to large vessel occlusion. World Neurosurgery, 114, e165-e172. https://doi.org/10. 1016/j.wneu.2018.02.126

27. Bellwald, S., et al. (2017). Direct mechanical intervention versus bridging therapy in stroke patients eligible for intravenous thrombolysis: A pooled analysis of 2 registries. Stroke, 48(12), 3282-3288. https://doi.org/10.1161/ STROKEAHA.117.018459.

28. Broeg-Morvay, A., et al. (2016). Direct mechanical intervention versus combined intravenous and mechanical intervention in large artery anterior circulation stroke: A matched-pairs analysis. Stroke, 47(4), 1037-1044. https:// doi.org/10.1161/STROKEAHA.115.011134.

29. Kaesmacher, J., et al. (2019). Direct mechanical thrombectomy in tPAineligible and -eligible patients versus the bridging approach: A metaanalysis. Journal of Neurointerventional Surgery, 11(1), 20-27. https://doi.org/ 10.1136/neurintsurg-2018-013834

30. Katsanos, A. H., \& Tsivgoulis, G. (2019). Is intravenous thrombolysis still necessary in patients who undergo mechanical thrombectomy? Current Opinion in Neurology, 32(1), 3-12. https://doi.org/10.1097/WCO. 0000000000000633.

31. Pan, X., Liu, G., Wu, B., Liu, X., \& Fang, Y. (2019). Comparative efficacy and safety of bridging strategies with direct mechanical thrombectomy in large vessel occlusion: A systematic review and meta-analysis. Medicine (Baltimore), 98(14), e14956. https://doi.org/10.1097/MD. 0000000000014956.

32. Arba, F., et al. (2017). Determinants of post-stroke cognitive impairment: Analysis from VISTA. Acta Neurologica Scandinavica, 135(6), 603-607. https:// doi.org/10.1111/ane.12637.

33. MoCA Montreal - Cognitive Assessment. [Online]. Available: https://www. mocatest.org/faq/. [Accessed: 04 Dec 2019].

34. Little, R. J. A., \& Rubin, D. B. (1989). The analysis of social science data with missing values. Sociological Methods \& Research, 18(2-3), 292-326. https:// doi.org/10.1177/0049124189018002004.

35. Cohen, J. (1988). Statistical power analysis for the behavioral sciences, (2nd ed. ). Hillsdale: L. Erlbaum Associates.

36. Little, R. J. A., \& Rubin, D. B. (2002). Statistical analysis with missing data.

37. Schafer, J. L. (1999). Multiple imputation: a primer. Statistical Methods in Medical Research, 8(1), 3-15. https://doi.org/10.1177/096228029900800102.

38. Broome, L. J., Battle, C. E., Lawrence, M., Evans, P. A., \& Dennis, M. S. (Dec. 2016). Cognitive outcomes following thrombolysis in acute ischemic stroke: A systematic review. Journal of Stroke and Cerebrovascular Diseases, 25(12), 2868-2875. https://doi.org/10.1016/j. jstrokecerebrovasdis.2016.07.048.

39. Laihosalo, M., Kettunen, J. E., Koivisto, A.-M., Dastidar, P., Ollikainen, J., \& Jehkonen, M. (2011). Thrombolytic therapy and visuoperceptual functions in right hemisphere infarct patients. Journal of Neurology, 258(6), 1021-1025. https://doi.org/10.1007/s00415-010-5873-0.

40. Pendlebury, S. T., Wadling, S., Silver, L. E., Mehta, Z., \& Rothwell, P. M. (2011). Transient cognitive impairment in TIA and minor stroke. Stroke, 42(11), 3116-3121. https://doi.org/10.1161/STROKEAHA.111.621490.

41. van Rooij, F. G., Kessels, R. P. C., Richard, E., De Leeuw, F.-E., \& van Dijk, E. J. (2016). Cognitive impairment in transient ischemic attack patients: A systematic review. Cerebrovascular Diseases, 42(1-2), 1-9. https://doi.org/10. $1159 / 000444282$.

42. Tang, E. Y., et al. (2018). Longitudinal Effect of Stroke on Cognition: A Systematic Review. Journal of the American Heart Association, 7(2). https:// doi.org/10.1161/JAHA.117.006443.

43. Liu, J., et al. (2017). Association between current smoking and cognitive impairment depends on age: A cross-sectional study in Xi'an, China. Medicina Clínica (Barcelona), 149(5), 203-208. https://doi.org/10.1016/j. medcli.2017.02.033.

44. Baumgart, M., Snyder, H. M., Carrillo, M. C., Fazio, S., Kim, H., \& Johns, H. (2015). Summary of the evidence on modifiable risk factors for cognitive decline and dementia: A population-based perspective. Alzheimers Dement, 11(6), 718-726. https://doi.org/10.1016/j.jalz.2015.05.016.

45. Graham, J. E., et al. (1997). Prevalence and severity of cognitive impairment with and without dementia in an elderly population. Lancet, 349(9068), 1793-1796. https://doi.org/10.1016/S01406736(97)01007-6.

46. Morley, J. E. (2018). An overview of cognitive impairment. Clinics in Geriatric Medicine, 34(4), 505-513. https://doi.org/10.1016/j.cger.2018.06. 003.

47. Harrison, J. K., Stott, D. J., McShane, R., Noel-Storr, A. H., Swann-Price, R. S., \& Quinn, T. J. (2016, 2016). Informant Questionnaire on Cognitive Decline in the Elderly (IQCODE) for the early diagnosis of dementia across a variety of healthcare settings. Cochrane Database of Systematic Reviews, (11). https:// doi.org/10.1002/14651858.CD011333.pub2. 
48. Pendlebury, S., Welch, S., Cuthbertson, F., Mariz, J., Mehta, Z., \& Rothwell, P. (2013). Telephone assessment of cognition after TIA and stroke: TICSm and telephone MoCA vs face-to-face MoCA and neuropsychological battery. Stroke, 44(1), 227-229. https://doi.org/10. 1161/STROKEAHA.112.673384.

49. Cohen, R. A., \& Alexander, G. E. (2017). Using TICS and T-MoCA for telephone assessments of vascular cognitive impairment: Promising call or put on hold? Stroke, 48(11), 2919-2921. https://doi.org/10.1161/STROKEAHA. 117.018828 .

\section{Publisher's Note}

Springer Nature remains neutral with regard to jurisdictional claims in published maps and institutional affiliations.

Ready to submit your research? Choose BMC and benefit from:

- fast, convenient online submission

- thorough peer review by experienced researchers in your field

- rapid publication on acceptance

- support for research data, including large and complex data types

- gold Open Access which fosters wider collaboration and increased citations

- maximum visibility for your research: over $100 \mathrm{M}$ website views per year

At $\mathrm{BMC}$, research is always in progress.

Learn more biomedcentral.com/submissions 